\title{
Sustained Activation of Guanylate Cyclase-A with TDT, a Natriuretic Peptide Derivative, Exhibits Cardiorenal Protection in Dahl Salt-Sensitive Hypertensive Rats $\$$
}

\author{
Shohei Oishi, Naoko Suzuki, Yuri Hasui, Tsuyoshi Homma, Masanori Obana, \\ Takahiro Nagayama, and Yasushi Fujio \\ Laboratory of Clinical Science and Biomedicine, Graduate School of Pharmaceutical Sciences, Osaka University, Osaka, Japan \\ (S.O., M.O., Y.F.); End-Organ Disease Laboratories (S.O., Y.H., T.H.), Rare Disease \& LCM Laboratories (T.N.), and Research \\ Function (N.S.), Daiichi Sankyo Co., Ltd, Tokyo, Japan
}

Received August 3, 2017; accepted October 2, 2017

\begin{abstract}
Heart failure often presents with prognosis-relevant impaired renal function. To investigate whether the chronic activation of guanylate cyclase-A (GC-A) protects both heart and kidney, we examined the effects of TDT, a neprilysin (NEP)-resistant natriuretic peptide (NP) derivative, on cardiac and renal dysfunction in Dahl salt-sensitive hypertensive (DS) rats. Pretreatment with NEP or NEP inhibitor did not influence GC-A activation by TDT both in vitro and in vivo, resulting in a long-acting profile of TDT compared with native human atrial NP (hANP). The repeated administration of TDT to DS rats suppressed the progress of cardiac hypertrophy, systolic/diastolic dysfunction, and proteinuria in a dose-dependent manner. Compared with vehicle and hANP, salt diet-induced podocyte injury was reduced by TDT, as analyzed by urinary podocalyxin concentration, renal
\end{abstract}

\section{Introduction}

Approximately 26 million people worldwide suffer from heart failure (HF), which is one of the major causes of death (Ambrosy et al., 2014). Blockade of neurohumoral systems, such as the renin-angiotensin-aldosterone system (RAAS) and the sympathetic nervous system (SNS), improves the prognosis of patients with HF significantly but its efficacy is still unsatisfactory. Patients with $\mathrm{HF}$, especially those caused by hypertension, have a high prevalence of prognosisrelevant comorbidities, such as chronic kidney disease. Accumulating evidence has demonstrated that interaction between the heart and kidney bidirectionally accelerates the progression of their pathologies, which are termed collectively as cardiorenal syndrome (Schefold et al., 2016). Therefore, therapeutic strategies against HF should have as their objective not simply cardioprotection but also renoprotection.

https://doi.org/10.1124/jpet.117.244459.

S This article has supplemental material available at jpet.aspetjournals.org. expression of nephrin mRNA, and glomerular expression of desmin protein. Since glomerular TRPC6 plays detrimental roles in podocyte homeostasis, we examined the renal expression of TRPC6 in DS rats and found that salt diet upregulated the expression of TRPC6. Importantly, TRPC6 induction was significantly decreased in TDT-treated rats, compared with vehicle and hANP. Consistently, in primary-culture podocytes from DS rats, TDT inhibited ATP-induced calcium influx, similar to TRPC inhibitor SKF96365. Finally, TDT-mediated protection of podocytes was abolished by protein kinase G inhibitor KT5823. In conclusion, TDT treatment attenuated heart and kidney dysfunction, accompanied by podocyte protection through inhibition of TRPC6. Thus, long-acting NPs could be a new avenue for treatment of heart failure.

ABBREVIATIONS: ANP, atrial natriuretic peptide; $\mathrm{CHF}$, chronic heart failure; $\mathrm{CHO}$, Chinese hamster ovary; DS, Dahl salt-sensitive hypertensive; DWS, diastolic wall strain; GC-A, guanylate cyclase-A; HF, heart failure; HR, heart rate; KT5823, PKG inhibitor VII; NEP, neprilysin; NGAL, neutrophil gelatinase-associated lipocalin; PKG, protein kinase G; SBP, systolic blood pressure; SKF96365, 1-[ $\beta$-[3-(4-methoxyphenyl)propoxy]-4methoxyphenethyl]-1H-imidazole; UP, urinary protein. 
Recent peptide engineering technology has structurally modified native NPs to prolong their half-lives by conferring resistance to enzymatic degradation by NEP (Meems and Burnett, 2016). In this study, TDT, an NEP-resistant derivative of taipan natriuretic peptide (TNP) (Alewood et al., 2008), was used to achieve sustained activation of GC-A. Considering that NEP inhibitor, in combination with angiotensin II receptor blocker, clinically improves the prognosis of CHF patients without worsening renal function (McMurray et al., 2014), it is probable that TDT could be a novel promising drug for CHF; however, its pharmacological characterization and medical benefits remain to be elucidated.

Here, we have hypothesized that sustained activation of GC-A could improve cardiac and renal disorders of HF that result from hypertension. And we examined the effects of sustained GC-A activation by TDT on cardiac and renal dysfunction using Dahl salt-sensitive hypertensive (DS) rats.

\section{Materials and Methods}

Peptides. TDT (SDSKIGNGCFGHKIDRINHVSNLGCNRIMQNPPKKFSGE, disulfide bond between C9-C25) was synthesized by Scrum Inc. (Tokyo, Japan) as described in patent no. US2008015374 (Alewood et al., 2008). The purity was confirmed by high-performance liquid chromatography analysis to be $>95 \%$. hANP was purchased from Daiichi Sankyo Co., Ltd. (Tokyo, Japan).

Animals. All experimental procedures were approved by the Institutional Animal Care and Use Committee of Daiichi Sankyo Co., Ltd. The investigation conformed to the Guide for the Care and Use of Laboratory Animals, 8th ed, updated by the U.S. National Research Council Committee in 2011.

In Vitro Human GC-A/cGMP Assay. The open reading frame of human GC-A (hGC-A) constructed in vector pCMV6-Entry (cat. no. RC209267) was purchased from OriGene Technologies, Inc. (Rockville, $\mathrm{MD})$. Chinese hamster ovary (CHO)-K1 cells were stably transfected with the plasmid DNA by Lipofectamine (Thermo Fisher Scientific, Waltham, MA) and selected by G418 (Thermo Fisher Scientific) using a limiting-dilution method to obtain a single clone. CHO/hGC-A stable clones were then screened for the best response to hANP.

For the cGMP assay, hGC-A-expressing or parental CHO cells were seeded in a 384-well plate at $4 \times 10^{3}$ cells/well and cultured for 1 day under $5 \% \mathrm{CO}_{2}$ at $37^{\circ} \mathrm{C}$. After removal of culture medium, the cells were incubated with 1.6 mM 1-methyl-3-isobutylxanthine (Merck Millipore, Billerica, MA) for 10 minutes followed by incubation for 15 minutes with hANP or TDT, which had been preincubated with or without $10 \mu \mathrm{g} / \mathrm{ml}$ of NEP (R\&D Systems, Minneapolis, MN) for 30 minutes at $37^{\circ} \mathrm{C}$. The cells were lysed, and then cGMP concentration in the lysate was determined using a cGMP assay kit (Cisbio, Codolet, France).

In Vivo Plasma cGMP Measurement. Plasma cGMP concentration was evaluated using 8-week-old male SD rats under isoflurane (2\%) anesthesia. hANP or TDT at $100 \mathrm{nmol} / \mathrm{kg}$ was administrated via bolus injection just after intravenous infusion of saline or phosphoramidon (Santa Cruz Biotechnology, Dallas, TX), an NEP inhibitor, at $825 \mathrm{nmol} / \mathrm{kg}$ per minute for 2 minutes, followed by continuous administration at $165 \mathrm{nmol} / \mathrm{kg}$ per minute until the end of the experiment, according to a previous report (Hashimoto et al., 1994). cGMP concentration in $\mathrm{Na}_{2}$ EDTA plasma at each time point was measured by Amersham cGMP Enzymeimmunoassay Biotrak (EIA) System (GE Healthcare, Little Chalfont, UK).

Experimental Protocol of Chronic Study. Male DS rats were purchased from Japan SLC, Inc. (Shizuoka, Japan). The rats were maintained in a room at $50 \%$ relative humidity (acceptable range: $30 \%-70 \%$ ) and $22^{\circ} \mathrm{C}$ (acceptable range: $19-25^{\circ} \mathrm{C}$ ) under a 12-hour light/dark cycle and allowed free access to diet (FR-2; Funabashi Farm, Chiba, Japan) and water.
At 7 weeks of age, rats began a high-salt diet (FR-2 containing $8 \%$ $\mathrm{NaCl}$; Funabashi Farm) and at 12 weeks of age were divided into four groups $(n=13-14)$ on the basis of body weight, systolic blood pressure (SBP), diastolic wall strain (DWS), left ventricular end-diastolic dimension, and urinary protein (UP) excretion. The vehicle (5\% glucose), hANP (40 nmol/kg), or TDT (low dose: $8 \mathrm{nmol} / \mathrm{kg}$ or high dose: $40 \mathrm{nmol} / \mathrm{kg}$ ) was subcutaneously administrated twice a day for 8 weeks up to 20 weeks of age. As a control, six rats were fed a normalsalt diet (FR-2 containing $0.3 \% \mathrm{NaCl}$ ).

Measurement of Systolic Blood Pressure and Heart Rate. SBP and heart rate (HR) were measured by the tail-cuff method using a noninvasive sphygmomanometer (BP-98A; Softron, Tokyo, Japan) under conscious, restrained conditions at 11 (before dosing), 16, and 19 weeks of age. Individual data are presented as the mean values of three consecutive measurements.

Measurement of Urinary and Blood Biochemical Parameters. Twenty-four-hour urine collection and blood sampling were performed at 11 and 18 weeks of age. Urinary protein concentration and plasma creatinine concentration were measured by an automated clinical analyzer (BiOLiS 24i Premium; Tokyo Boeki Machinery, Tokyo, Japan) and urinary electrolytes were detected by an electrolyte analyzer (STAX-2; Techno Medica, Kanagawa, Japan). Urinary podocalyxin (Excocell, Philadelphia, PA) and kidney injury molecule1 (KIM-1; R\&D systems) were determined by ELISA kits.

Echocardiography. High-resolution transthoracic echocardiography was performed using Vevo 2100 equipped with a 13-MHz linear array transducer (Fujifilm Visualsonics, Toronto, ON, Canada) under isoflurane (1.5-3\%) anesthesia at 11 and 19 weeks of age, as described previously (Kumagai et al., 2016). Administration of test peptides was stopped on the day of the measurement. According to the method of Takeda et al. (2009), DWS was calculated as an indicator of myocardial wall stiffness using the parameters of LV posterior wall thickness at end-systole (LVPWs) and at end-diastole (LVPWd) using the following equation:

\section{DWS $=1-$ LVPWs $/$ LVPWd}

Hemodynamic Measurements. The hemodynamic study was performed at 20 weeks of age under $2 \%$ isoflurane anesthesia using a catheter pressure transducer (Millar Instruments, Houston, TX) according to a previously described method (Klotz et al., 2006). The

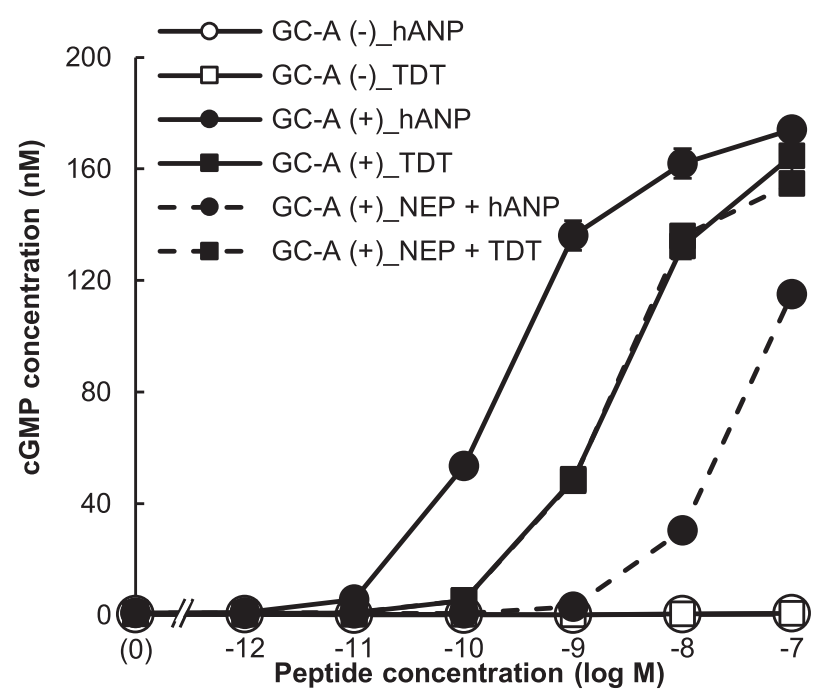

Fig. 1. TDT induces cGMP production via GC-A in an NEP-resistant manner. CHO cells stably expressing human GC-A were incubated with the indicated concentrations of peptides for 15 minutes after preincubation with or without NEP for 30 minutes. cGMP concentrations were measured with a Cisbio HTRF-based assay. Representative data are shown from three independent experiments that were performed in quadruplicate. Data are presented as the mean \pm S.E.M. Error bars that do not show are smaller than symbols. 
A

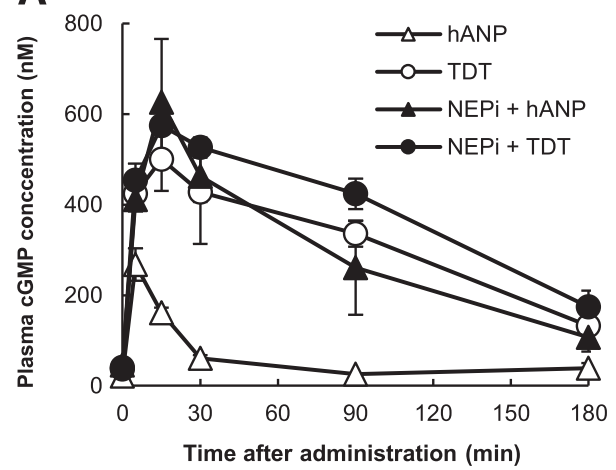

B

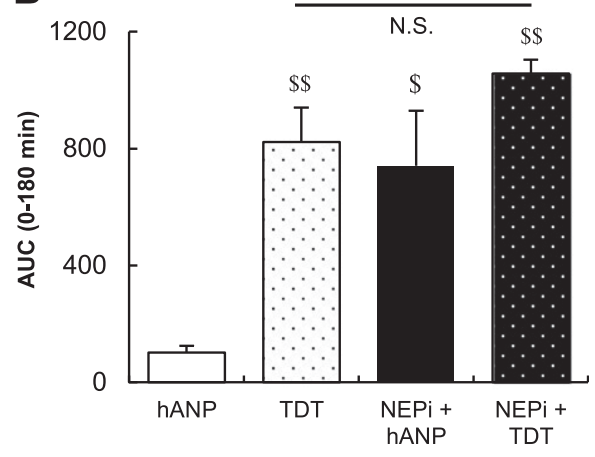

Fig. 2. TDT administration enhances plasma cGMP concentration independently of NEP activity in rats. (A) After bolus injection of TDT or hANP at $100 \mathrm{nmol} / \mathrm{kg}$ in the presence or absence of phosphoramidon, an NEP inhibitor (NEPi), plasma concentrations of cGMP were measured. (B) AUC of the change in plasma cGMP concentration from baseline is shown. Data are presented as the mean \pm .M. S.E.M $\left(n=3\right.$, each). ${ }^{\$} P<0.05$, $\$ \$ P<0.01$ vs. hANP-treated group. time constant of LV isovolumic pressure decline, tau, was calculated using the Glantz method (Raff and Glantz, 1981).

Histopathological Evaluation. The heart samples, fixed in $10 \%$ neutral buffered-formaldehyde solution, were embedded in paraffin. Sections were stained with hematoxylin and eosin. Cross-sectional areas of cardiomyocytes were measured by a researcher who was blinded to the experimental conditions, as the mean value of 100 eosin-positive cardiomyocytes of the papillary muscle, using morphometric analysis software (analySIS; Soft Imaging System, Münster, Germany) in a randomly selected microscope field in each section.
Quantitative PCR. Relative gene expression in the kidney was determined by quantitative real-time polymerase chain reaction (PCR) using TaqMan Gene Expression Assays and TaqMan universal PCR master mix (Thermo Fisher Scientific) according to a general procedure (Arai et al., 2016). The following assays were used: B cell leukemia/lymphoma 2 (Bcl2; Rn99999125_m1), collagen 1a1 (Col1a1; Rn01463848_m1), interleukin-1 $\beta$ (IL-1 $\beta$; Rn00580432_m1), nephrin (Nphs1; Rn00674268_m1), neutrophil gelatinase-associated lipocalin (NGAL; Lcn2; Rn00590612_m1), and glyceraldehyde-3-phosphate dehydrogenase (GAPDH; Rn99999916_s1). PCR reactions were performed using a 7900 HT Fast Real Time PCR system (Thermo Fisher
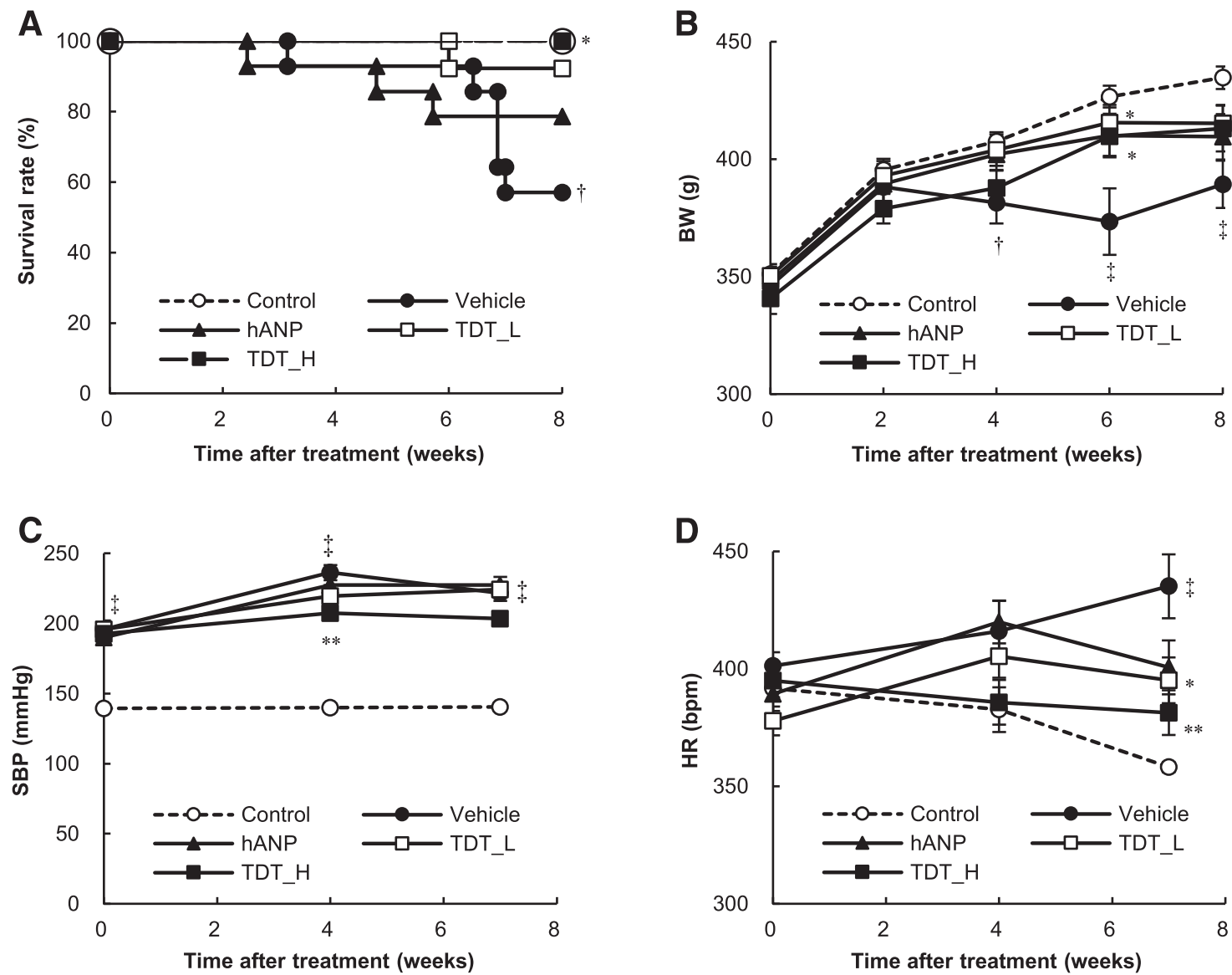

Fig. 3. Effects of chronic TDT therapy on survival, body weight, systolic blood pressure, and heart rate in DS rats. DS rats were fed a high-salt diet (8\% $\mathrm{NaCl}$ ) from 7 weeks of age. hANP or TDT was twice-daily subcutaneously dosed from 12 weeks of age for 8 weeks. Control: normal diet ( $0.3 \%$ NaCl)-fed group $(n=6)$. Vehicle: vehicle-treated group $(n=14)$. hANP: hANP $(40 \mathrm{nmol} / \mathrm{kg})$-treated group $(n=14)$. TDT_L: TDT ( $8 \mathrm{nmol} / \mathrm{kg})$-treated group $(n=13)$. TDT_H: TDT (40 nmol/kg)-treated group $(n=13)$. (A) Survival rate. (B) Body weight. (C) SBP. (D) HR. Data are presented as the mean \pm S.E.M. Error bars that do not show are smaller than symbols. ${ }^{\dagger} P<0.05,{ }^{\ddagger} P<0.01$ vs. control; ${ }^{*} P<0.05,{ }^{* *} P<0.01$ vs. vehicle. 
Scientific). Relative mRNA levels of each gene were calculated by normalizing them to GAPDH mRNA level.

Glomerular Protein Expression. The kidney samples, fixed in $10 \%$ neutral buffered-formaldehyde solution, were embedded in paraffin and then sectioned. Glomerular expression of TRPC6 and desmin was evaluated by semiquantitative scoring of immunohistostained samples. Immunohistostaining was performed with an EnVision+ Kit (Agilent, Santa Clara, CA) in accordance with the procedure described by the manufacturer. Rabbit anti-rat TRPC6 (1: 100 dilution, ACC-120; Alomone Laboratories, Jerusalem, Israel) and mouse anti-rat desmin (1:400 dilution; D33; Abcam, Cambridge, UK) antibodies were used as primary antibodies (Nijenhuis et al., 2011; Sonneveld et al., 2013). Glomerular TRPC6 and desmin expression was scored semiquantitatively from 0 to 5 on the basis of the extent of immunostaining intensity in 20 randomly selected glomeruli per kidney (negative $=0,1-20 \%$ positive with mild staining $=1,1-20 \%$ positive with intense staining or $21-40 \%$ positive with mild staining $=2,21-40 \%$ positive with intense staining or $41-60 \%$ positive with mild staining $=3,41-60 \%$ positive with intense staining or $61-80 \%$ positive with mild staining $=4$, and $61-100 \%$ positive with intense staining $=5$ ). Scoring was performed independently by two investigators who were blinded to the experimental conditions.

Primary Cultured Rat Podocytes. Eighteen- to 21-week-old DS rats were fed an $8 \% \mathrm{NaCl}$-containing diet from 7 weeks of age. Podocytes were isolated according to the conventional sieving method (Chen et al., 2006) with minor modification. In brief, renal cortical tissue homogenates were strained through steel sieves with pore sizes in the order of 250,150 , and then $75 \mu \mathrm{m}$. The glomeruli retained on the top of the $75-\mu \mathrm{m}$ sieve were washed and resuspended in ice-cold culture medium (Dulbecco's modified Eagle's medium/F-12 containing $5 \%$ fetal bovine serum supplemented with $0.5 \%$ insulin-transferrinselenium-A, and $100 \mathrm{IU} / \mathrm{ml}$ penicillin-streptomycin). The glomeruli were then plated on collagen I-coated 10-cm dishes. After 4 days' culture in $5 \% \mathrm{CO}_{2}$ at $37^{\circ} \mathrm{C}$, outgrowing glomerular cells were trypsinized and passed over a $40-\mu \mathrm{m}$ nylon strainer. Only cells passing through the strainer were replated on collagen I-coated plates and cultured for 1 day. The cultured cells mostly consisted of arborized or cobble stone-like podocytes under the light microscope.

Intracellular Calcium Measurements. Cultured rat podocytes, seeded at $3 \times 10^{4}$ cells/well in a 96 -well plate for 1 day, were washed twice with phosphate-buffered saline and then suspended in RPMI 1640 medium with or without $1 \mu$ M KT5823 (Merck Millipore), a PKG inhibitor, or $10 \mu \mathrm{M}$ SKF-96365 (Merck Millipore), a TRPC inhibitor. Ninety minutes after the incubation, cells were treated with $10 \mu \mathrm{M}$ Fura 2-AM (Thermo Fisher Scientific) and incubated for 30 minutes followed by wash and treatment with $100 \mathrm{nM}$ TDT dissolved in Krebs-

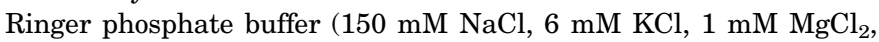
$10 \mathrm{mM}$ D-glucose, and $10 \mathrm{mM}$ HEPES) containing $1.5 \mathrm{mM} \mathrm{CaCl}_{2}$ for 15 minutes. The culture plates were mounted on an inverted microscope stage and exposed to 340- and 380-nm wavelength light using a multiple wavelengths high-solution fluorescence microscopy system (IonOptix, Westwood, MA). The arborized podocytes were then selected and monitored. To stimulate calcium entry into the cells, $10 \mu \mathrm{M}$ ATP (Merck Millipore) was added. Data were presented as the fluorescence ratio $340 / 380 \mathrm{~nm}$.

Statistical Analysis. Data are shown as means \pm S.E.M. Statistical analyses between two groups were performed by Student's or Welch's $t$ test. For multiple comparisons, the Dunnett's test or Tukey's test was performed depending on the purpose. Survival analysis was performed using Kaplan-Meier analysis and the log-rank test. A value of $P<0.05$ was considered to be statistically significant. All statistical analyses were performed using SAS System Release 9.2 (SAS Institute, Tokyo, Japan).

\section{Results}

TDT Activated GC-A in an NEP-Resistant Manner. To assess activation of GC-A by TDT, we measured the concentrations of cGMP in human GC-A (hGC-A)-expressing cells after stimulation with TDT or hANP (Fig. 1). TDT showed a full agonistic activity to hGC-A relative to hANP in a concentration-dependent manner, though the $\mathrm{EC}_{50}$ value
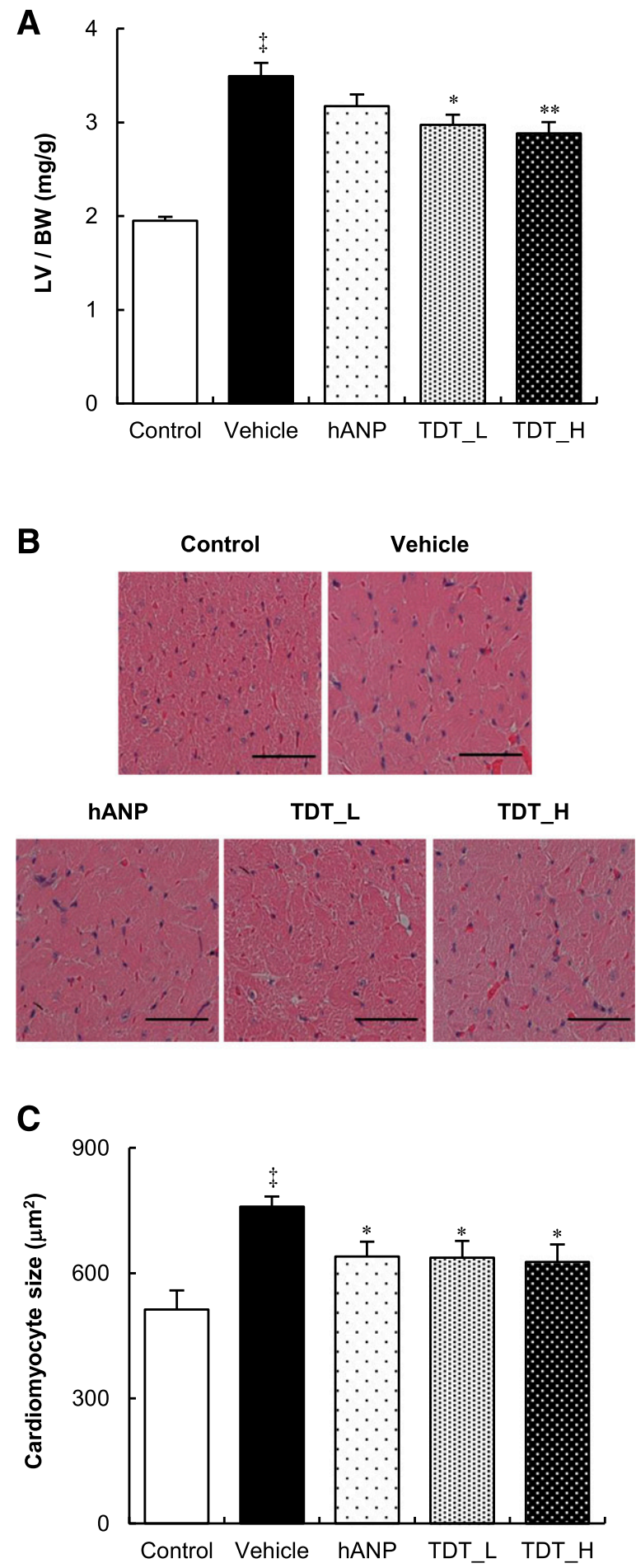

Fig. 4. Chronic TDT therapy inhibited cardiac hypertrophy in DS rats. Rats were fed a high-salt diet, concomitant with treatment of hANP or TDT. (A) The left ventricular weight (LV)/body weight (BW) ratio was measured ( $n=6$ for control, $n=13-14$ for the other groups). (B) Representative images of hematoxylin and eosin-stained sections of the left ventricle are shown (scale bar, $50 \mu \mathrm{m}$ ). (C) Cross-sectional areas of cardiomyocytes were measured $(n=4$ for control, $n=8$ for the other groups). Data are presented as the mean \pm S.E.M. ${ }^{\ddagger} P<0.01$ vs. control; ${ }^{*} P<0.05,{ }^{* *} P<0.01$ vs. vehicle. 
of TDT was higher than that of hANP (TDT, $195 \mathrm{pM}$; hANP, $19 \mathrm{pM})$. Importantly, preincubation of hANP with NEP reduced hGC-A activation by hANP, but not that of TDT.

To evaluate the activation of GC-A with TDT in vivo, TDT or hANP was intravenously administered and plasma cGMP concentrations were measured. Plasma cGMP concentrations were more potently elevated and more slowly reduced by TDT than by hANP (Fig. 2A). Consistent with in vitro experiments, NEP inhibition by phosphoramidon enhanced AUC of cGMP after hANP administration up to a level similar to TDT alone, but did not significantly influence AUC after TDT treatment (Fig. 2B).

Chronic Treatment of TDT Ameliorated Salt-Induced Hypertensive Heart Failure in DS Rats. To address the beneficial therapeutic properties of TDT, DS rats were fed a high-salt diet and mortality, body weight, blood pressure, and heart rate were analyzed. Kaplan-Meier analyses revealed that the salt diet reduced the survival rate in DS rats and that mortality was improved by the treatment with TDT, compared with vehicle (Fig. 3A). In this experiment, we used two dosages of TDT and confirmed that TDT improved salt-induced mortality in a dose-dependent manner. Only one animal in 14 animals in TDT at low dosage (TDT_L) group died through the experiment, whereas 6 of 14 in vehicle group died. Considering that TDT at high dosage (TDT_H) perfectly improved survival, TDT_L seemed to be effective, but statistical significance between TDT_L and vehicle groups was not observed, probably because animals died concentratedly from 6 to 8 weeks in the vehicle group. Consistent with the increased mortality, body weight gradually decreased in vehicle-treated rats, whereas body weight increased in TDTtreated animals with a significant difference at week 6 (Fig. 3B). SBP was significantly elevated by the high-salt diet at the initiation of treatment with NPs, compared with control. Subsequent salt loading continued to elevate SBP comparably in vehicle, hANP, and TDT_L group, which was suppressed by TDT_H at week 4 (Fig. 3C). Compared with the control, the high-salt diet increased HR in the vehicletreated group, but TDT significantly reduced HR at week 6 (Fig. 3D).

The high-salt diet induced cardiac hypertrophy with an increase of the left ventricle-to-body weight (LV/BW) ratio, which was reduced by TDT in a dose-dependent manner (Fig. 4A). Likewise, histologic analyses demonstrated that the increase in cardiomyocyte size induced by the high-salt diet was reduced by hANP and TDT (Fig. 4B and 4C).
To examine the effects of TDT on cardiac function, echocardiographic analyses (Table 1) and hemodynamic assessments (Table 2) were performed. Echocardiographic examination demonstrated that the high-salt diet reduced fractional shortening, ejection fraction, stroke volume, cardiac output, and DWS, an indicator of ventricular wall stiffness, and that both TDT_L and TDT_H attenuated the impairment of these parameters. Consistently, hemodynamic analyses using a catheter showed that both systolic $\left(\mathrm{dP} / \mathrm{dt}_{\max } / \mathrm{P}\right)$ and diastolic $\left(\mathrm{dP} / \mathrm{dt}_{\min } / \mathrm{P}\right.$ and $\left.\mathrm{Tau}\right)$ functions were worsened by the high-salt diet and that TDT ameliorated these functions. Of note, measurement by catheter method demonstrated that hANP and TDT_L did not lower BP, whereas TDT_H significantly reduced $\mathrm{BP}$, consistent with the data by tail cuff method.

TDT Protected Renal Disorder Induced by a HighSalt Diet in DS Rats. Renal function was analyzed 6 weeks after NP treatment (Table 3). UP excretion was increased in the high-salt diet groups compared with the normal-diet group at the initiation of NP therapy (data not shown). UP excretion progressively increased $>10$ times in the vehicle group, compared with the control group. Importantly, TDT suppressed the increase in UP excretion induced by the highsalt diet. Although the sodium excretion/intake ratio was not influenced by NP treatment, each peptide potently enhanced creatinine clearance with statistical significance compared with the vehicle-treated group. To support these findings, we determined renal mRNA expression of inflammatory cytokine IL-1 $\beta$, profibrotic marker collagen 1a1, and antiapoptotic marker Bcl-2. Salt overload upregulated IL-1 $\beta$ and collagen $1 \mathrm{a} 1$, and downregulated Bcl-2, whereas hANP and TDT significantly inhibited these changes (Supplemental Figure).

Podocyte and Tubular Injury Were Suppressed by TDT. Given the antiproteinuric effect of TDT, we next evaluated injury markers of tubules and podocytes, which play a pivotal role in the glomerular filtration barrier. Urinary Kim-1 excretion and mRNA expression of NGAL in the kidney were attenuated by TDT in a dose-dependent manner, indicating that hANP and TDT ameliorated tubular injury (Fig. 5A and B). Urinary excretion of podocalyxin, a component of the slit diaphragm in podocytes, was remarkably higher in the vehicle-treated group than in the control group, whereas both TDT_L and TDT_H, but not hANP, significantly suppressed the elevation (Fig. 5C). Consistently, mRNA expression of nephrin, an important component of the renal filtration barrier, was upregulated by TDT in a dose-dependent manner (Fig. 5D) but not by hANP. Consistent with these results,

TABLE 1

TDT preserved cardiac systolic and diastolic function in DS rats

Cardiac systolic/diastolic functional parameters were measured by echocardiography at 19 weeks of age $(n=6$ for control,

$n=11$ for vehicle and hANP, $n=12$ for TDT_L, and $n=13$ for TDT_H). Data are presented as the mean \pm S.E.M $n=11$ for vehicle and hANP, $n=12$ for TDT_L, and $n=13$ for TDT_H). Data are pre
${ }^{*} P<0.05,{ }^{\sharp} P<0.01$ vs. control group; ${ }^{*} P<0.05,{ }^{*} P<0.01$ vs. vehicle-treated group.

\begin{tabular}{lccccc}
\hline & Control & Vehicle & hANP & \multicolumn{1}{c}{ TDT_L } & TDT_H \\
\hline LVDd $(\mathrm{mm})$ & $7.50 \pm 0.24$ & $7.73 \pm 0.31$ & $7.88 \pm 0.16$ & $7.69 \pm 0.21$ & $7.84 \pm 0.20$ \\
IVSd $(\mathrm{mm})$ & $2.16 \pm 0.13$ & $2.84 \pm 0.08^{\ddagger}$ & $2.59 \pm 0.08^{*}$ & $2.61 \pm 0.08$ & $2.35 \pm 0.10^{* *}$ \\
LVPWd $(\mathrm{mm})$ & $2.07 \pm 0.09$ & $2.62 \pm 0.10^{\ddagger}$ & $2.45 \pm 0.05$ & $2.55 \pm 0.07$ & $2.32 \pm 0.5^{*}$ \\
FS $(\%)$ & $41.0 \pm 1.2$ & $29.2 \pm 3.3^{\dagger}$ & $34.9 \pm 2.0$ & $40.4 \pm 2.5^{* *}$ & $44.8 \pm 1.7^{* *}$ \\
EF $(\%)$ & $70.1 \pm 1.5$ & $53.0 \pm 4.6^{\dagger}$ & $61.9 \pm 2.9$ & $68.6 \pm 3.0^{* *}$ & $74.0 \pm 1.9^{* *}$ \\
SV $(\mu \mathrm{l})$ & $210 \pm 14$ & $161 \pm 6^{*}$ & $204 \pm 8^{* *}$ & $214 \pm 10^{* *}$ & $244 \pm 13^{* *}$ \\
CO $(\mathrm{ml} / \mathrm{min})$ & $84.9 \pm 5.3$ & $62.4 \pm 3.0^{\ddagger}$ & $78.7 \pm 3.1^{* *}$ & $81.6 \pm 4.2^{* *}$ & $93.2 \pm 4.9^{* *}$ \\
DWS & $0.328 \pm 0.032$ & $0.191 \pm 0.021^{*}$ & $0.262 \pm 0.018$ & $0.272 \pm 0.017^{* *}$ & $0.348 \pm 0.011^{* *}$ \\
\hline
\end{tabular}

CO, cardiac output; EF, ejection fraction; FS, fractional shortening; IVSd, intraventricular septum in diastole; LVDd, left ventricular diameter in diastole; LVPWd, left ventricular posterior wall in diastole; SV, stroke volume. 
TABLE 2

TDT improved hemodynamics in DS rats

Hemodynamic parameters were measured by catheter with a pressure transducer at 20 weeks of age $(n=5$ for control, $n=7$ for vehicle, $n=6$ for hANP, $n=9$ for TDT_L, $n=9$ for TDT_H). Data are presented as the mean \pm S.E.M. ${ }^{\dagger} P<0.05,{ }^{\star} P<0.01$ vs. control group; ${ }^{*} P<0.05$, ** $P<0.01$ vs. vehicle-treated group.

\begin{tabular}{|c|c|c|c|c|c|}
\hline & Control & Vehicle & hANP & TDT_L & TDT_H \\
\hline $\mathrm{SBP}(\mathrm{mmHg})$ & $138 \pm 4$ & $236 \pm 3^{\ddagger}$ & $226 \pm 5$ & $227 \pm 7$ & $206 \pm 6^{* *}$ \\
\hline $\mathrm{DBP}(\mathrm{mmHg})$ & $97 \pm 4$ & $172 \pm 2^{\ddagger}$ & $164 \pm 4$ & $165 \pm 5$ & $149 \pm 5^{* *}$ \\
\hline $\mathrm{dP} / \mathrm{dt}_{\max }(\mathrm{mmHg} / \mathrm{sec})$ & $8099 \pm 402$ & $10519 \pm 767$ & $9987 \pm 451$ & $12195 \pm 714$ & $11144 \pm 625$ \\
\hline $\mathrm{dP} / \mathrm{dt}_{\max } / \mathrm{P}(/ \mathrm{sec})$ & $98.7 \pm 3.0$ & $78.2 \pm 5.2^{\dagger}$ & $82.5 \pm 2.5$ & $92.9 \pm 4.1^{*}$ & $93.9 \pm 1.9^{*}$ \\
\hline $\mathrm{dP} / \mathrm{dt}_{\min }(\mathrm{mmHg} / \mathrm{sec})$ & $-9706 \pm 652$ & $-9073 \pm 582$ & $-8971 \pm 702$ & $-10917 \pm 511^{*}$ & $-11594 \pm 346^{* *}$ \\
\hline $\mathrm{dP} / \mathrm{dt}_{\min } / \mathrm{P}(/ \mathrm{sec})$ & $-101.1 \pm 4.4$ & $-51.7 \pm 4.1^{\dagger}$ & $-59.6 \pm 4.2$ & $-68.5 \pm 5.4^{*}$ & $-76.9 \pm 3.6^{* *}$ \\
\hline LVEDP (mmHg) & $9.5 \pm 1.1$ & $13.1 \pm 2.6$ & $11.3 \pm 1.5$ & $10.0 \pm 1.6$ & $9.3 \pm 1.5$ \\
\hline Tau (msec) & $12.0 \pm 0.6$ & $24.8 \pm 3.8^{\dagger}$ & $20.2 \pm 1.4$ & $18.5 \pm 1.4$ & $16.3 \pm 0.9^{*}$ \\
\hline
\end{tabular}

DBP, diastolic blood pressure; LVEDP, left ventricular end diastolic pressure.

immunohistological analyses using anti-desmin antibody demonstrated that glomerular desmin expression, an established podocyte injury marker, was less remarkable in the TDT-treated groups compared with the vehicle- and hANPtreated groups (Fig. 5E and F). Taken together, TDT ameliorated both tubular and podocyte injury. Since TDT more potently protected from podocyte injury than did hANP, and a recent report suggested that GC-A plays pivotal roles in podocyte homeostasis, we focused on the podocytes.

TDT Reduced Glomerular TRPC6 Expression in a Dose-Dependent Manner. To address the key renoprotective mechanism of TDT, we examined the glomerular protein expression level of TRPC6, since the upregulation of TRPC6 expression results in the impairment of podocyte homeostasis (Moller et al., 2007). As depicted in Fig. 6, TDT reduced TRPC6 expression elevated by salt overloading in a dose-dependent manner. Importantly, TDT_L exhibited inhibitory effects on TRPC6 induction more remarkably than hANP, suggesting that TDT could be a promising renoprotective drug.

To examine whether TDT directly transduces its signals in podocytes, we stimulated cultured rat podocytes with TDT and measured cGMP production (Fig. 7A). TDT stimulation promoted cGMP production, suggesting that TDT could preserve podocyte homeostasis directly. Since TRPC6-mediated calcium influx deteriorates podocyte homeostasis (Winn et al., 2005), we tested the effect of TDT on intracellular calcium influx evoked by ATP with or without SKF-96365 and KT5823 in primary rat podocytes (Fig. 7B and C). Robust calcium influx induced by ATP was largely inhibited by pretreatment with SKF-96365, a TRPC inhibitor, indicating that calcium influx was largely mediated by TRPC. TDT reduced calcium influx, and KT5823, a PKG inhibitor, completely abolished the effect of TDT, suggesting that TDT regulated TRPC function through PKG. Importantly the treatment with SKF-96365 in combination with TDT did not exert additional inhibition compared with SKF-96365 alone, indicating that TDT inhibited calcium influx mainly by inhibiting TRPC.

\section{Discussion}

HF is one of the major causes of death. Since the NEP inhibitor in combination with angiotensin receptor blocker improves the prognosis of HF, we hypothesized that NEPresistant NPs could prevent HF progression. In the present study, we examined the effects of TDT, an NEP-resistant NP, on cardiac and renal dysfunction in DS rats. The repeated dosing of TDT ameliorated high-salt diet-induced cardiac hypertrophy and suppressed systolic and diastolic dysfunction. In addition to cardioprotective effects, TDT attenuated proteinuria induced by the high-salt diet. Moreover, TDT suppressed podocyte injury associated with the downregulation of TRPC6 expression. Interestingly, TDT directly activated GC-A and reduced calcium influx by inactivating TRPC6 activity in podocyte. Thus, we propose that there are protective effects of chronic treatment with NEP-resistant NPs as a long-acting GC-A activator against cardiac and renal disorders.

Entresto, a combination drug of the NEP inhibitor sacubitril and angiotensin receptor blocker valsartan, is clinically used as a novel anti-CHF drug to reduce the risk of cardiovascular death and hospitalization (McMurray et al., 2014). The cardioprotective effects of NEP inhibition are thought to be explained largely by the elevation of endogenous NPs (Solomon et al., 2012). However, since NEP inactivates a wide range of peptide hormones and secretory factors in addition to NPs (Campbell, 2017), it was difficult to make clear whether exogenous NP treatment could ameliorate CHF. Despite recent advances in peptide engineering technology and

TABLE 3

TDT improved renal function in DS rats

Urine collection for 24 hours was performed at 18 weeks of age. Data are presented as the mean \pm S.E.M. ${ }^{\dagger} P<0.05$, ${ }^{\dagger} P<0.01$ vs. control group; $* P<0.05, * * P<0.01$ vs. vehicle-treated group.

\begin{tabular}{|c|c|c|c|c|c|}
\hline & Control $(n=6)$ & Vehicle $(n=10)$ & hANP $(n=11)$ & TDT_L $(n=12)$ & TDT_H $(n=13)$ \\
\hline UV (ml/day) & $13.1 \pm 3.0$ & $95.9 \pm 9.2^{\ddagger}$ & $88.5 \pm 8.6$ & $76.5 \pm 5.4$ & $78.5 \pm 5.7$ \\
\hline Water intake (g/day) & $35 \pm 3$ & $110 \pm 9$ & $105 \pm 8$ & $94 \pm 6$ & $96 \pm 6$ \\
\hline UP/day (mg/day) & $27 \pm 3$ & $308 \pm 32^{\ddagger}$ & $277 \pm 45$ & $236 \pm 32$ & $190 \pm 32 *$ \\
\hline $\mathrm{Na}^{+}$excretion/intake (g/g) & $0.58 \pm 0.09$ & $0.31 \pm 0.01^{\dagger}$ & $0.32 \pm 0.01$ & $0.33 \pm 0.01$ & $0.32 \pm 0.01$ \\
\hline $\mathrm{CCr}(\mathrm{ml} / \mathrm{min})$ & $2.0 \pm 0.4$ & $2.4 \pm 0.3$ & $3.5 \pm 0.4^{*}$ & $3.6 \pm 0.3^{*}$ & $4.2 \pm 0.3^{* *}$ \\
\hline
\end{tabular}

CCr, creatinine clearance; UV, urine volume. 
A

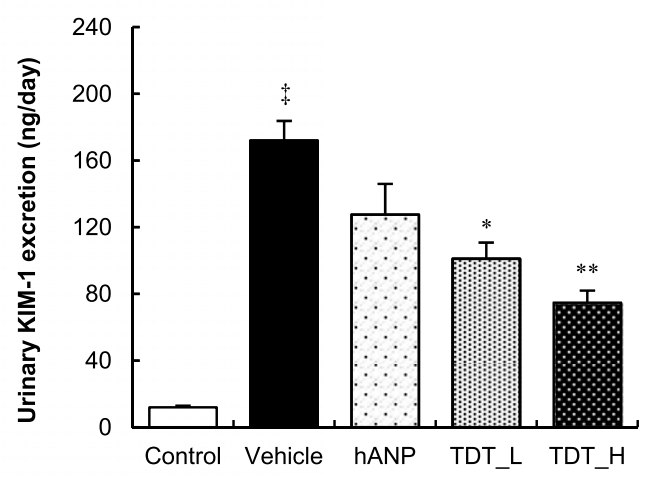

C

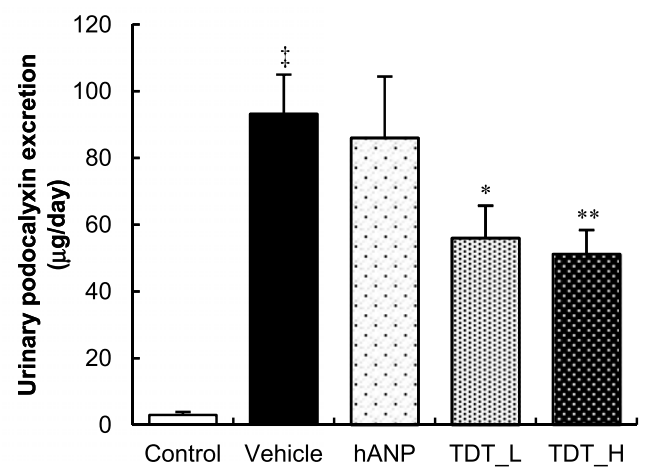

$\mathbf{E}$

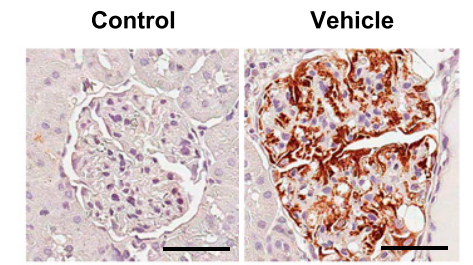

hANP

TDT_L

TDT_H

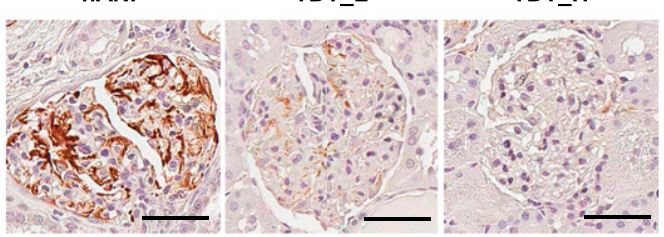

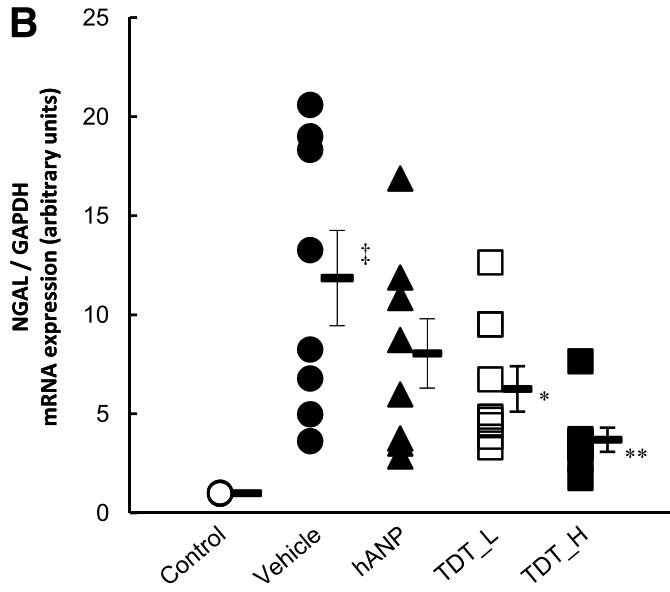

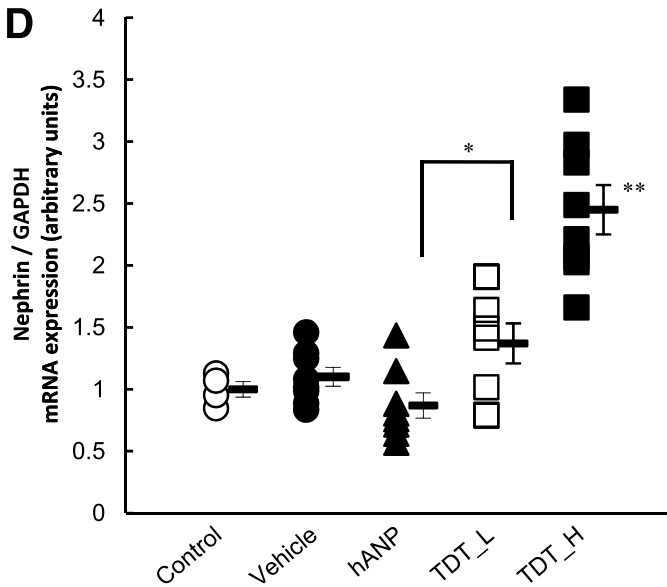

$\mathbf{F}$

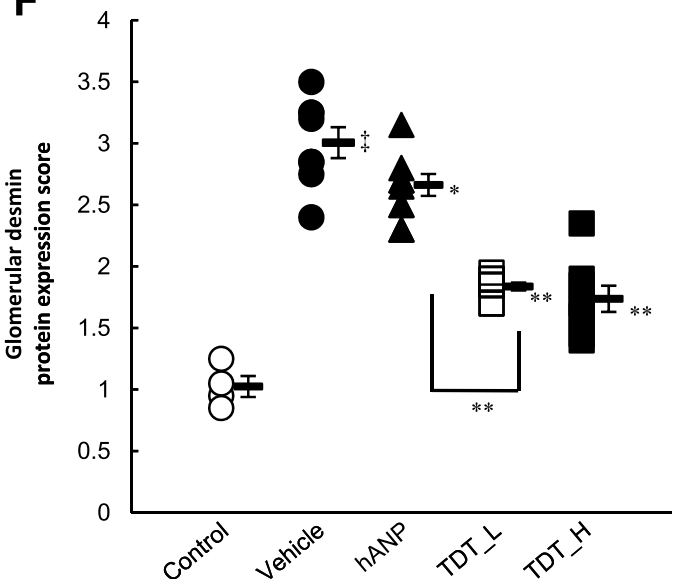

Fig. 5. TDT, but not hANP, protected tubules and podocytes from high salt-induced injury in DS rats. (A and B) Tubular injury markers and (C-F) podocyte injury markers were investigated. (A) Urinary excretion of KIM-1, an injury marker of proximal tubules, and (C) that of podocalyxin, an indicator of podocyte detachment and fragmentation, were measured at 18 weeks of age ( $n=5$ for control, $n=10$ for vehicle, $n=11$ for hANP, $n=12$ for TDT LL, and $n=13$ for TDT_H). (B) mRNA expression of NGAL, another indicator of tubular injury, and (D) that of nephrin, a component of podocytes, were determined by real-time quantitative PCR in the DS rat kidneys at 20 weeks of age and normalized to the GAPDH mRNA level $(n=4$ for control, $n=8$ for the other groups). ( $\mathrm{E}$ and F) Glomerular protein expression of the podocyte injury marker desmin was evaluated by immunohistochemical analysis. (E) Representative images are shown (scale bar, $50 \mu \mathrm{m}$ ). (F) Desmin protein expression was semiquantitatively shown $(n=4$ for control, $n=8$ for the other groups). Data are presented as the mean \pm S.E.M. ${ }^{\star} P<0.01$ vs. control; ${ }^{*} P<0.05,{ }^{* *} P<0.01$ vs. vehicle or indicated group.

success in half-life extension of native NPs, there have been very few reports to reveal the therapeutic potential of their chronic application. Here, we focused on TDT as a novel NEP- resistant NP. TDT is an engineered NP derivative from taipan natriuretic peptide, which is isolated from the venom of the inland taipan (Alewood et al., 2008). Since TDT was resistant 
A
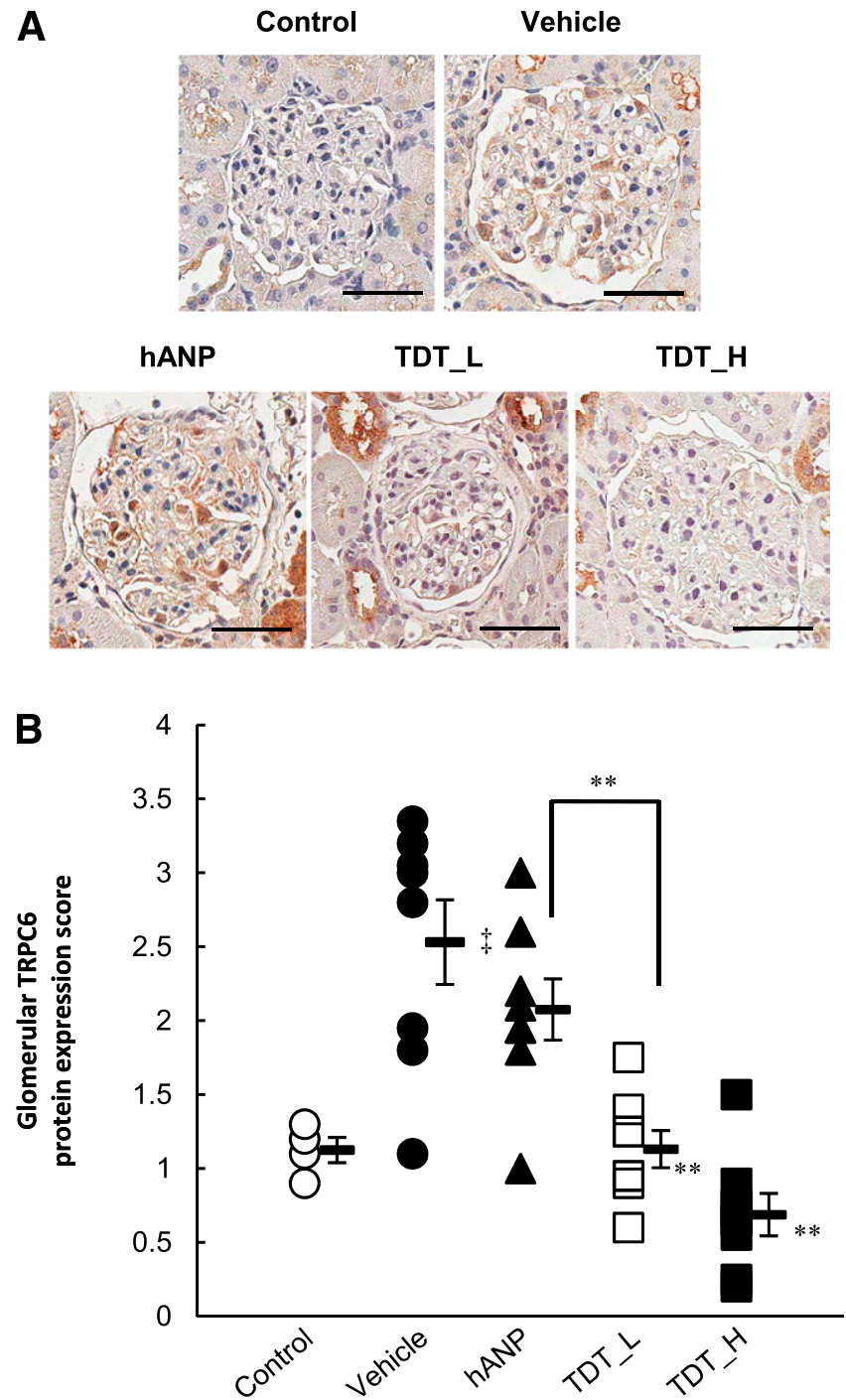

Fig. 6. TDT reduced glomerular expression of TRPC6 protein in DS rats. Glomerular expression of TRPC6 protein in DS rats at 20 weeks of age was semiquantitatively determined by immunohistochemical analysis. (A) Representative images are shown (Scale bar, $50 \mu \mathrm{m}$ ). (B) Glomerular expression of TRPC6 protein was semiquantitatively shown ( $n=4$ for control, $n=8$ for the other groups). Data are presented as the mean \pm S.E.M. ${ }^{*} P<0.01$ vs. control, ${ }^{*} * P<0.01$ vs. vehicle or indicated group.

to degradation by NEP and exhibited cardiorenal protective effects, we could propose that the NEP-resistant NP would be a novel therapeutic strategy, at least for HF patients intolerant of NEP inhibitors. In addition, considering that the upregulation of endogenous NPs by NEP inhibitor is theoretically limited, exogenous NPs could additionally activate GC-A. Further studies would be required to make clear whether TDT shows clinical benefits additive to NEP inhibitors.

Recent studies using genetically modified mice showed that podocyte-specific ablation of the NP receptor GC-A gene results in increased susceptibility to renal injury (Staffel et al., 2017), indicating that GC-A in podocytes play important roles in renal protection; however, it remains to be fully elucidated whether pharmacological activation of the NP receptor protects kidneys from chronic stress, because conventional NPs are rapidly degraded by NEP. Here, using TDT, we successfully demonstrated that chronic activation of GC-A
A

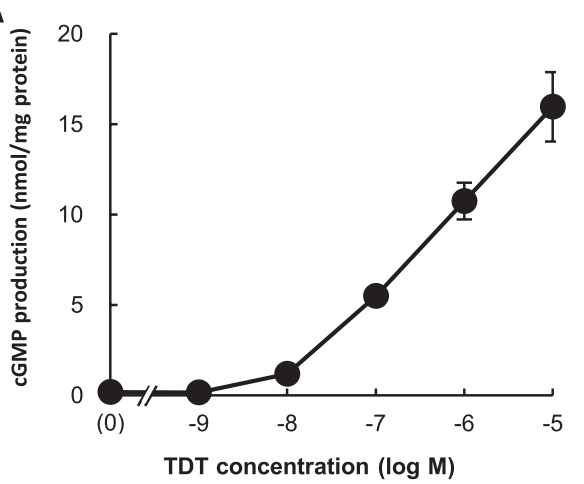

B

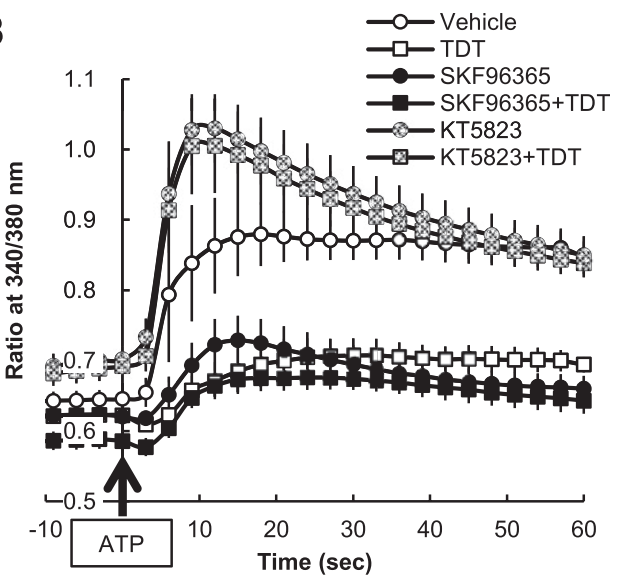

C

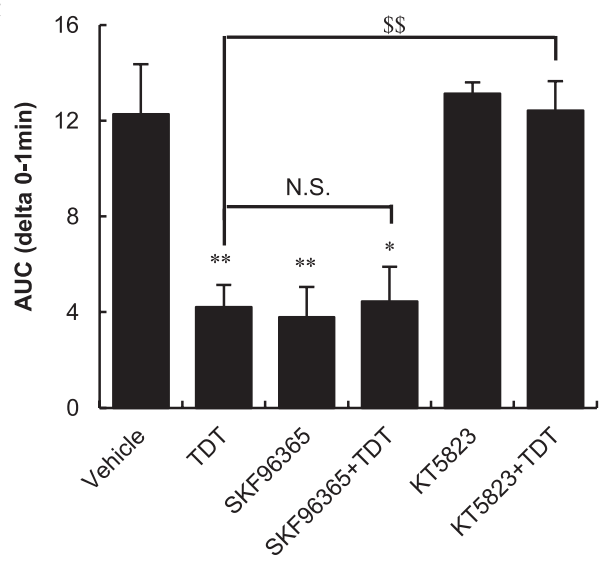

Fig. 7. TDT inhibited TRPC-mediated calcium influx through PKG in primary cultured rat podocytes. (A) Primary cultured podocytes from DS rats were incubated with the indicated concentrations of TDT for 25 minutes. cGMP concentrations were then measured and normalized by protein amounts. Data are from four independent experiments performed in duplicate. (B and C) Cultured rat podocytes were pretreated with $100 \mathrm{nM}$ TDT with or without TRPC inhibitor SKF96365 $(10 \mu \mathrm{M})$ or PKG inhibitor KT5823 $(1 \mu \mathrm{M})$ for 30 minutes, followed by exposure to $10 \mu \mathrm{M}$ ATP to evoke $\mathrm{Ca}^{2+}$ influx. Intracellular $\mathrm{Ca}^{2+}$ concentration was determined by Fura-2 ratiometry $(n=4-5)$. (B) $\mathrm{Ca}^{2+}$ influx was measured at the indicated time points. (C) AUC of the change in calcium influx from the baseline was calculated. Data are presented as the mean \pm S.E.M. Error bars that do not show are smaller than symbols. $* P<0.05$, ** $P<$ 0.01 vs. vehicle group; ${ }^{\$} P<0.01$ vs. indicated group.

by long-acting NPs prevented renal dysfunction accompanied by protection of podocytes. Most importantly, TDT protected podocytes even at a low dose that did not significantly impact blood pressure, glomerular filtration rate, and natriuresis. Considering the limitation of the dosing amount of GC-A 
activator because of its potent hypotensive effect, TDT could be a novel, well-balanced renoprotective agent with respect to efficacy and safety.

However, to mention direct organ protection by TDT, there are two major limitations in this study. First, the measuring methods of blood pressure, tail-cuff and intra-arterial catheter, could mask the changes in BP, since animals were subjected to immobilization and anesthetic stress. To assess chronic effect of TDT on BP in a condition closer to physiologic settings, continuous recording of $\mathrm{BP}$ in free-moving animals would be needed. Second, it should be noted that our results presented here do not necessarily claim superiority of TDT over hANP, because hANP was used at a single dose. To compare the effectiveness of cardiac and renal protection between TDT and hANP adequately, analysis of pharmacokinetic/pharmacodynamic relationships using extra dosing amounts will be needed. In addition, we demonstrated that TDT protected not only podocytes but also tubules, even at a low dose. Consistently, Kato et al. (2017) reported that systemic GC-A KO mice are more susceptible to renal damage than podocyte-specific GC-A KO mice in a blood pressure-independent manner, which indicates the importance of other renal cells, including tubules, in the signaling of NPs. Further investigations regarding the direct protection of tubules by GC-A activation will be required.

Glomerulosclerosis is caused by nuclear factor of activated T cells (NFAT)-mediated induction of TRPC6 (Nijenhuis et al., 2011). As NFAT is activated by calcineurin in a calciumdependent manner, it is probable that TRPC-mediated calcium influx induces TRPC6 expression as a positive feedback mechanism. In this context, it is important that TDT treatment reduced TRPC activity both in vitro and in vivo, contributing to the prevention of glomerulosclerosis. Interestingly, we also demonstrated that TRPC-mediated calcium influx was inhibited by PKG inhibitor, consistent with the previous report that the renal dysfunction by $G C$ - $A$ gene ablation is attenuated by a TRPC inhibitor (Staffel et al., 2017).

In conclusion, we demonstrated that TDT exhibited cardiorenal protection in DS rats. The generation of NEP-resistant NP using peptide engineering technology could be a promising strategy against cardiorenal diseases.

\section{Acknowledgments}

The authors thank Shin Nippon Biomedical Laboratories, Ltd., Drug Safety Research Laboratories (Kagoshima, Japan) for their expertly conducted experiments.

\section{Authorship Contributions}

Participated in research design: Oishi, Nagayama, Fujio.

Conducted experiments: Oishi, Suzuki, Hasui.

Performed data analysis: Oishi, Homma.

Wrote or contributed to the writing of the manuscript: Oishi, Obana, Fujio.

\section{References}

Alewood P, Head GA, and Fry BG (2008) inventors, The University Of Queensland, Baker Heart Research Institute, assignee. Proteinaceous compounds and uses therefor. US patent US20,080,153,747. 2008 Jun 26

Ambrosy AP, Fonarow GC, Butler J, Chioncel O, Greene SJ, Vaduganathan M, Nodari S, Lam CS, Sato N, Shah AN, et al. (2014) The global health and economic burden of hospitalizations for heart failure: lessons learned from hospitalized heart failure registries. $J$ Am Coll Cardiol 63:1123-1133.

Arai K, Morikawa Y, Ubukata N, Tsuruoka H, and Homma T (2016) CS-3150, a novel nonsteroidal mineralocorticoid receptor antagonist, shows preventive and therapeutic effects on renal injury in deoxycorticosterone acetate/salt-induced hypertensive rats. J Pharmacol Exp Ther 358:548-557.

Campbell DJ (2017) Long-term neprilysin inhibition - implications for ARNIs. Nat Rev Cardiol 14:171-186.

Chen CA, Hwang JC, Guh JY, Tsai JC, and Chen HC (2006) TGF-beta1 and integrin synergistically facilitate the differentiation of rat podocytes by increasing alphasmooth muscle actin expression. Transl Res 148:134-141.

Hashimoto Y, Nakao K, Hama N, Imura H, Mori S, Yamaguchi M, Yasuhara M, and Hori R (1994) Clearance mechanisms of atrial and brain natriuretic peptides in rats. Pharm Res 11:60-64.

Kato Y, Mori K, Kasahara M, Osaki K, Ishii A, Mori KP, Toda N, Ohno S, Kuwabara T, Tokudome T, et al. (2017) Natriuretic peptide receptor guanylyl cyclase-A pathway counteracts glomerular injury evoked by aldosterone through p38 mitogen-activated protein kinase inhibition. Sci Rep 7:46624.

Kishimoto I, Tokudome T, Horio T, Garbers DL, Nakao K, and Kangawa K (2009) Natriuretic peptide signaling via guanylyl cyclase (GC)-A: an endogenous protective mechanism of the heart. Curr Cardiol Rev 5:45-51.

Klotz S, Hay I, Zhang G, Maurer M, Wang J, and Burkhoff D (2006) Development of heart failure in chronic hypertensive Dahl rats: focus on heart failure with preserved ejection fraction. Hypertension 47:901-911.

Kumagai S, Nakayama H, Fujimoto M, Honda H, Serada S, Ishibashi-Ueda H, Kasai A, Obana M, Sakata Y, Sawa Y, et al. (2016) Myeloid cell-derived LRG attenuates adverse cardiac remodelling after myocardial infarction. Cardiovasc Res 109: $272-282$.

McMurray JJ, Packer M, Desai AS, Gong J, Lefkowitz MP, Rizkala AR, Rouleau JL Shi VC, Solomon SD, Swedberg K, et al.; PARADIGM-HF Investigators and Committees (2014) Angiotensin-neprilysin inhibition versus enalapril in heart failure. N Engl J Med 371:993-1004.

Meems LMG and Burnett JC, Jr (2016) Innovative therapeutics: designer natriuretic peptides. JACC Basic Transl Sci 1:557-567.

Möller CC, Wei C, Altintas MM, Li J, Greka A, Ohse T, Pippin JW, Rastaldi MP, Wawersik S, Schiavi S, et al. (2007) Induction of TRPC6 channel in acquired forms of proteinuric kidney disease. J Am Soc Nephrol 18:29-36.

Nijenhuis T, Sloan AJ, Hoenderop JG, Flesche J, van Goor H, Kistler AD, Bakker M, Bindels RJ, de Boer RA, Möller CC, et al. (2011) Angiotensin II contributes to podocyte injury by increasing TRPC6 expression via an NFAT-mediated positive feedback signaling pathway. Am J Pathol 179:1719-1732.

Raff GL and Glantz SA (1981) Volume loading slows left ventricular isovolumic relaxation rate. Evidence of load-dependent relaxation in the intact dog heart. Circ Res 48:813-824.

Schefold JC, Filippatos G, Hasenfuss G, Anker SD, and von Haehling S (2016) Heart failure and kidney dysfunction: epidemiology, mechanisms and management. Nat Rev Nephrol 12:610-623.

Seidelmann SB, Vardeny O, Claggett B, Yu B, Shah AM, Ballantyne CM, Selvin E MacRae CA, Boerwinkle E, and Solomon SD (2017) An NPPB promoter polymorphism associated with elevated N-terminal pro-B-type natriuretic peptide and lower blood pressure, hypertension, and mortality. J Am Heart Assoc 6.

Solomon SD, Zile M, Pieske B, Voors A, Shah A, Kraigher-Krainer E, Shi V, Bransford T, Takeuchi M, Gong J, et al.; Prospective comparison of ARNI with ARB on Management Of heart failUre with preserved ejectioN fracTion (PARAMOUNT) Investigators (2012) The angiotensin receptor neprilysin inhibitor LCZ696 in heart failure with preserved ejection fraction: a phase 2 double-blind randomised controlled trial. Lancet 380:1387-1395.

Sonneveld R, Ferrè S, Hoenderop JG, Dijkman HB, Berden JH, Bindels RJ, Wetzels JF, van der Vlag J, and Nijenhuis T (2013) Vitamin D down-regulates TRPC6 expression in podocyte injury and proteinuric glomerular disease. Am J Pathol 182:1196-1204.

Staffel J, Valletta D, Federlein A, Ehm K, Volkmann R, Füchsl AM, Witzgall R, Kuhn $\mathrm{M}$, and Schweda F (2017) Natriuretic peptide receptor guanylyl cyclase-A in podocytes is renoprotective but dispensable for physiologic renal function. $J \mathrm{Am}$ Soc Nephrol 28:260-277.

Takeda Y, Sakata Y, Higashimori M, Mano T, Nishio M, Ohtani T, Hori M Masuyama T, Kaneko M, and Yamamoto K (2009) Noninvasive assessment of wall distensibility with the evaluation of diastolic epicardial movement. J Card Fail 15: 68-77.

Volpe M (2014) Natriuretic peptides and cardio-renal disease. Int $J$ Cardiol 176: 630-639.

Winn MP, Conlon PJ, Lynn KL, Farrington MK, Creazzo T, Hawkins AF, Daskalakis N, Kwan SY, Ebersviller S, Burchette JL, et al. (2005) A mutation in the TRPC6 cation channel causes familial focal segmental glomerulosclerosis. Science 308: 1801-1804.

Address correspondence to: Dr. Yasushi Fujio, Laboratory of Clinical Science and Biomedicine, Graduate School of Pharmaceutical Sciences, Osaka University, Osaka, Japan 1-6 Yamadaoka, Suita, Osaka 565-0871, Japan. E-mail: fujio@phs.osaka-u.ac.jp. Or, Shohei Oishi, End-Organ Disease Laboratories, Daiichi Sankyo Co., Ltd, 1-2-58 Hiromachi, Shinagawa-ku, Tokyo 1408710, Japan. E-mail: oishi.shohei.pr@daiichisankyo.co.jp 